\title{
Microinductive Signal Conditioning With Resonant Differential Filters: High-Sensitivity Biodetection Applications
}

\author{
Salvatore Baglio, Alejandro Pérez-Rodríguez, Susanna Martínez, Christophe Serre, \\ Joan Ramon Morante, Jaume Esteve, and Josep Montserrat
}

\begin{abstract}
Inductive-based devices integrated with Si technology for biodetection applications are characterized, using simple resonant differential filter configurations. This has allowed the corroboration of the viability of the proposed circuits, which are characterized by their very high simplicity, for microinductive signal conditioning in high-sensitivity sensor devices. The simulation of these simple circuits predicts sensitivities of the differential output voltage which can achieve values in the range of $0.1-1 \mathrm{~V} / \mathrm{nH}$, depending on the coil parameters. These very high-sensitivity values open the possibility for the experimental detection of extremely small inductance changes in the devices. For real microinductive devices, both series resistance and parasitic capacitive components contribute to the decrease of the differential circuit sensitivity. Nevertheless, measurements performed using microcoils fabricated with relatively high series resistance and coupling parasitic effects have allowed detection of changes in the range of $2 \mathrm{nH}$, which are compatible with biodetection applications with estimated detection limits below the picomolarity range.
\end{abstract}

Index Terms-Detection of magnetic particles, integrated magnetic immunobiosensors, microcoil simulation, microinductive devices, signal conditioning.

\section{INTRODUCTION}

$\mathbf{I}$ MMUNOMAGNETIC biosensors based on the use of superparamagnetic particles (MPs) as the biological markers have received strong interest for the detection and quantification of biological substances [1]-[3]. This interest is motivated by their potential for the development of systems with very high sensitivity, low cost, and high specificity. Among the different sensor strategies, different groups have proposed the use of inductive-based devices, where sensing is determined by the changes in the inductance of a solenoid due to the presence of the MPs in the sample under analysis [1], [4]-[6]. In relation to other magnetic immunoassays, these biosensors are characterized by their very high simplicity, since in this case, the application of permanent magnetic fields is not necessary

Manuscript received July 26, 2006; revised February 14, 2007.

S. Baglio is with the Dipartimento Elettrico, Elettronico e Sistemistico, University of Catania, 95125 Catania, Italy.

A. Pérez-Rodríguez, S. Martínez, C. Serre, and J. R. Morante are with Electronic Materials and Engineering/Centre of Reference of Advanced Materials for Energy/Centre for Engineering of Microsystems, Department of Electrònica, Universitat de Barcelona, 08028 Barcelona, Spain.

J. Esteve and J. Montserrat are with Centre Nacional de MicroelectrònicaCSIC, Campus UAB, 08193 Bellaterra, Spain.

Color versions of one or more of the figures in this paper are available online at http://ieeexplore.iee.org.

Digital Object Identifier 10.1109/TIM.2007.895593 for sensor operation. This simplifies the design of the device and its integration in the whole system.

The potential of these inductive devices for the development of high-sensitivity sensors is strongly conditioned to the availability of a suitable signal conditioning circuit. One of the most used approaches is based on the measurement of the resonant frequency of an $L C$ circuit containing the inductive sensor. However, for very high-sensitivity applications, this approach is compromised by the stability required for the oscillator circuit. This has lead Kriz et al. [1], [4] to use a modified Maxwell bridge in their sensor configuration. This is based on the measurement of the magnetic permeability of a sample formed by silica particles, which wear MPs that are immobilized on their surface through antigen-antibody-antigen sandwich complexes involving the analyte. This system has allowed the achievement of a detection limit of $250 \mathrm{nM}$ using Concanavalin A as the target analyte.

According to Richardson et al. [5], [6], an improvement of the sensor sensibility requires the development of conditioning circuits alternative to these classical bridge topologies. These authors have developed a circuit with a voltage-controlled oscillator and a phase-locked loop detector to determine the resonant frequency of an $L C$ circuit, including the sensor solenoid. This allows overcoming the limitations that are related to the stability required for the oscillator. The sensor proposed uses a plastic strip where the MPs are immobilized at the end of the assay, and the amount of bounded MPs on the surface of the strip is determined by measuring the change in the inductance of the measuring coil when the strip is placed in the coil nucleus. This has allowed detection down to $10^{5}$ MPs that are immobilized on the strip surface. The system has been applied to the detection of human transferrin, obtaining a linear response with femtomolar detection limit (260 fM).

On the other hand, the integration of inductive devices in $\mathrm{Si}$ technology has several potential advantages as compared to the macroscopic solenoids. These include features related to the fabrication cost, yield, and reproducibility of the planar inductive devices. In addition, the miniaturization of the device dimensions will lead to a significant decrease of the volume of the sample under analysis, with a consequent expected decrease in the cost of the reagents per analysis. This also opens interesting perspectives for the development of more complex systems such as sensor matrices for the analysis of different components or sensors integrated in complex micrototal analysis 


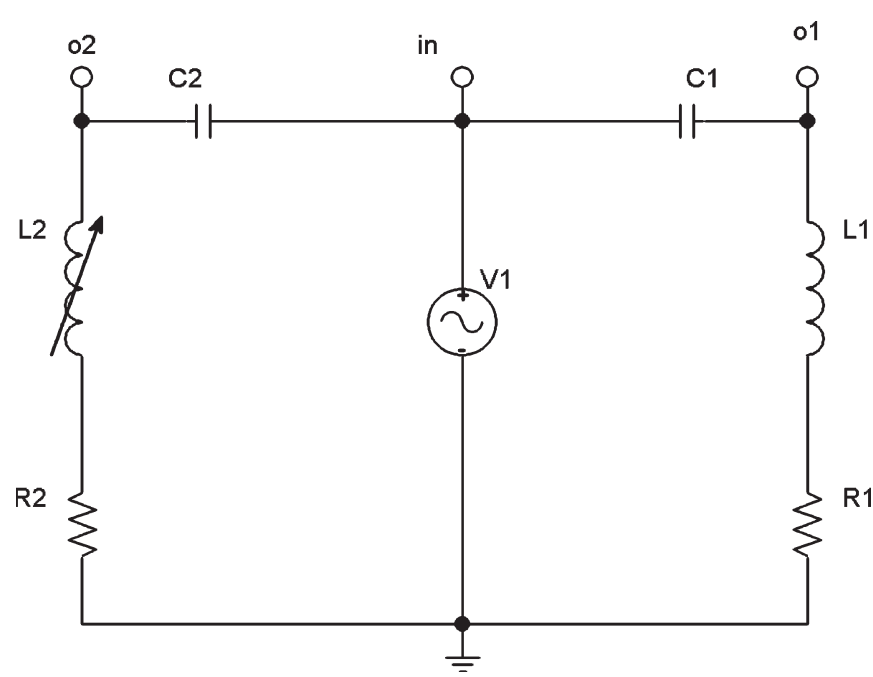

Fig. 1. Schematic of the differential filter.

systems. However, with respect to the currently available inductive sensors based on macroscopic solenoids, microinductances are characterized by the existence of a much higher series resistance due to intrinsic technological limitations when depositing the metal tracks using thin film technologies. This disables the use of the conditioning circuits developed using parallel $L C$ circuits, since the resistance considerably degrades the resonant peak.

An alternative interesting approach for the signal conditioning of the inductive sensor device is proposed by Diaz de Lezama et al. [7], which analyzes the use of very simple passive filter circuits. The comparative study of different circuit configurations has lead to an optimum design (in terms of both sensitivity and linearity) based on a second-order high pass filter. The simulation of this circuit leads to an overall maximum sensitivity of $8.86 \mathrm{~V} / \mathrm{mH}$.

In this framework, this paper analyzes the viability of simple second-order filter circuit topologies for sensor signal conditioning of microinductive devices. In contrast with the previous developments of Diaz de Lezama et al. [7], the requirements related to the high sensitivity lead to the design of differential configurations working at conditions close to resonance [8]. These circuits are applied to the characterization of microinductive coils, which are incorporated into passive filters made with discrete elements on a printed circuit board (PCB). The analysis of the fabricated prototypes has allowed demonstration of the ability of these very simple circuits for the high-sensitivity detection of the inductive changes from integrated microcoils, in spite of the high series resistance of the devices.

Following is a note about chemical units: Molarity $\mathrm{M}$ $(=\mathrm{mol} / \mathrm{l})$ is a standard and very convenient concentration unit, which is widely used in chemistry. Molarity is the number of moles of solute dissolved in one liter of solution (not per liter of solvent). Molarity is usually denoted with an italicized capital $\mathrm{M}$, e.g., a $0.50 \mathrm{M}$ solution.

\section{Circuit Description and Simulation}

The adopted circuit (Fig. 1) is based on two identical coils, where $L 1$ is a reference passive inductance (with series re-

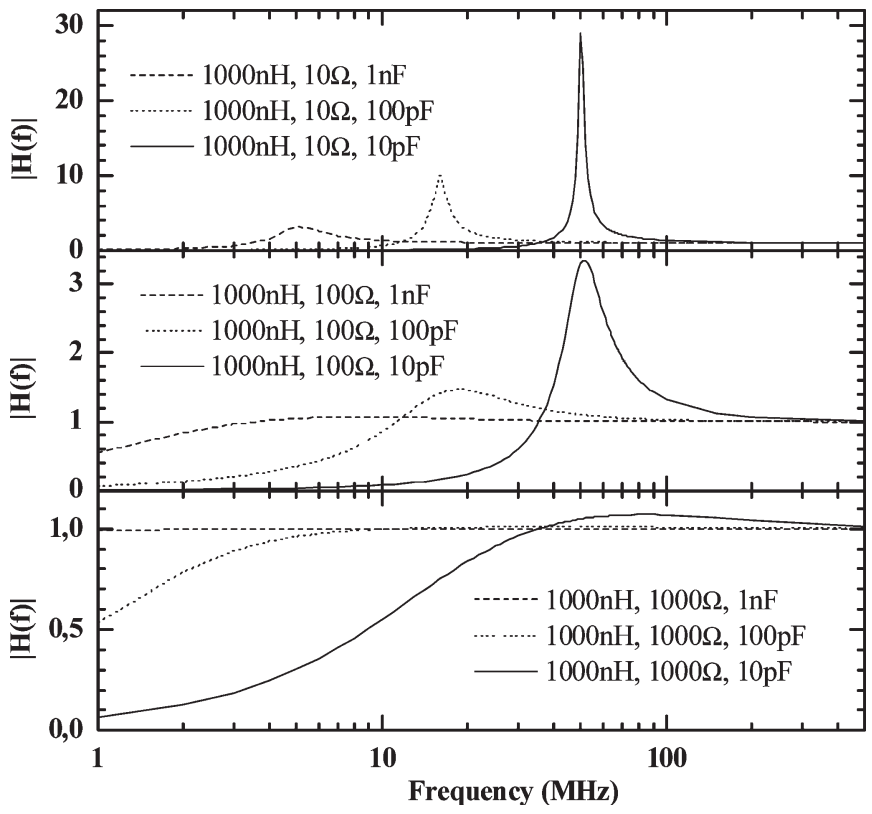

Fig. 2. Magnitude of the transfer function of one of the circuit branches versus frequency calculated with $L=1000 \mathrm{nH}$ and different combinations of $R(10-1000 \Omega)$ and $C(1 \mathrm{nF}-10 \mathrm{pF})$.

sistance $R 1$ ), and $L 2$ is the sensor inductance (with series resistance $R 2$ ).

This gives two similar filters connected in parallel, and the difference between the signals $V 01$ and $V 02$ is analyzed versus the inductance of the sensor $L 2$. The transfer function of each branch is given by

$$
H(s)=\frac{s^{2}+(R / L) s}{s^{2}+(R / L) s+(1 / C L)}
$$

which corresponds to a high-pass second-order resonant filter with quality factor $Q=\left(L \omega_{\mathrm{o}} / R\right)$ and natural angular frequency $\omega_{\mathrm{o}}=(1 / L C)^{1 / 2}$. Depending on the parameters of the circuit, this function has a resonant peak at a frequency $f_{\mathrm{p}}$, which is given by the expression

$$
f_{\mathrm{p}}=\left(f_{\mathrm{o}} / \sqrt{2}\right)\left(1+\sqrt{1+\frac{1}{2}\left(R / f_{\mathrm{o}} \pi L\right)^{2}}\right)^{1 / 2} .
$$

Fig. 2 shows the representation of the module of the transfer function versus the frequency calculated with $L=1000 \mathrm{nH}$ and using different combinations of $R$ (between 10 and $1000 \Omega$ ) and $C$ (between $1 \mathrm{nF}$ and $10 \mathrm{pF}$ ).

This figure clearly illustrates the strong dependence of the shape and position of the resonant peak on the electrical parameters of the circuit. For the given values of $L$ and $R$, a decrease of the capacity $C$ leads to an increase of the intensity of the peak, which becomes narrower, as well as an increase in $f_{\mathrm{p}}$. On the other hand, the shape of the peak shows a similar dependence on the resistance $R$ : As $R$ increases, the intensity of the peak decreases, and its width increases. The increase of $R$ may even lead to the disappearance of the circuit resonance. For the values used in Fig. 2, this occurs for $R=100 \Omega, C=1 \mathrm{nF}$, and for $R=1000 \Omega, C \geq 100 \mathrm{pF}$. 


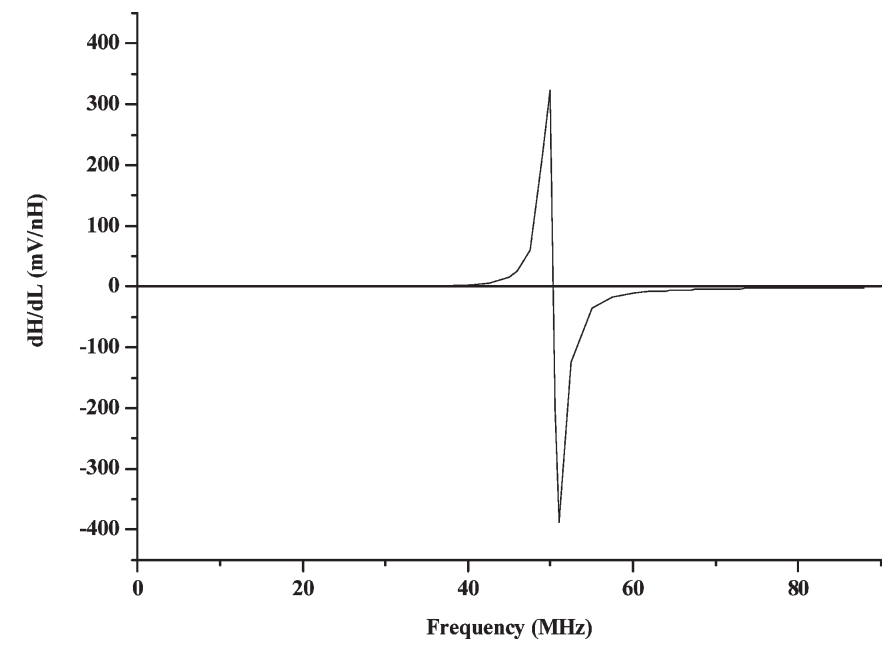

Fig. 3. $d|H(f)| / d L$ for a filter with $L=1000 \mathrm{nH}, C=10 \mathrm{pF}$, and $R=10 \Omega$.

In principle, if both branches of the circuit have the same values of $R, C$, and $L$, the corresponding transfer functions will be identical, and this will result in a null differential output. Then, a change in the value of $L 2$ would determine a change in the transfer function of the corresponding branch, giving rise to a differential output voltage. According to this, the sensitivity of the circuit to the changes of $L 2$ is determined by the sensitivity of the magnitude of the transfer function on the value of $L$, which is given by its derivative $d|H(f)| / d L$. This parameter is plotted in Fig. 3, for a filter with $L=1000 \mathrm{nH}, C=10 \mathrm{pF}$, and $R=10 \Omega$. For these values, expression (2) gives a resonant frequency $f_{\mathrm{p}}=50.34 \mathrm{MHz}$.

As shown in Fig. 3, the sensitivity of the magnitude of the transfer function on the change of $L$ is maximum for frequencies close to $f_{\mathrm{p}}$.

The results of the simulation of the differential circuit versus the value of $L 2$ are plotted in Fig. 4. This simulation has been performed with a standard circuit simulator (PSpice, [14]), and assuming $V_{\text {in }}=1 \mathrm{~V}$ and $L 1=1000 \mathrm{nH}, R 1=R 2=$ $10 \Omega, C 1=C 2=10 \mathrm{pF}$, with values of $L 2$ between 1000 and $1100 \mathrm{nH}$. Working with a frequency close to $f_{\mathrm{p}}=50.34 \mathrm{MHz}$ gives a differential output sensitivity of $S=339 \mathrm{mV} / \mathrm{nH}$, with a linear behavior for inductance variations $\Delta L 2 \leq 30 \mathrm{nH}$. This represents an improvement of more than four orders of magnitude for the circuit sensitivity with respect to that previously reported by Diaz de Lezama et al. [7] working with a single branch nonresonant circuit.

A potential drawback of this circuit is the relatively high value of the resonant frequency $f_{\mathrm{p}}$. Decreasing the working frequency below this value leads to conditions away from resonance, which determines a strong decrease of the sensitivity as well as a degradation of the circuit linearity. An alternative to this is to increase the value of the capacitance in the circuit. In relation to the simulation shown in Fig. 4, an increase of two orders of magnitude in the value of $C$ (up to $1 \mathrm{nF}$ ) leads to a decrease in about one order of magnitude of $f_{\mathrm{p}}$ (down to about 5.15 MHz). However, as shown in Fig. 2, this also gives a decrease and broadening of the resonance peak (1). The decrease in the intensity of the resonant peak leads to a concomitant decrease in the sensitivity of the differential circuit

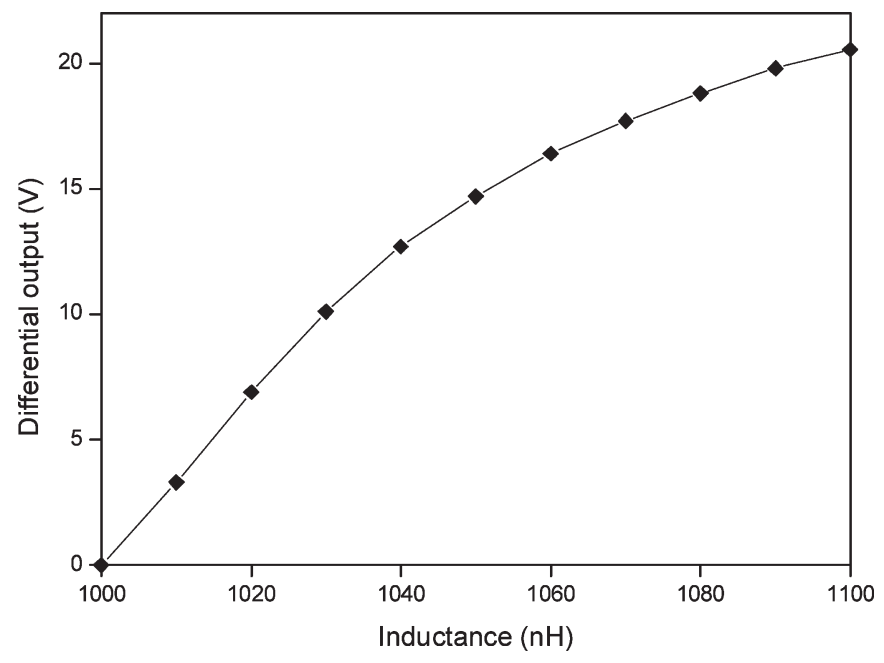

Fig. 4. Simulation of the differential output of the circuit from Fig. 1 ( $L=$ $1000 \mathrm{nH}, R=10 \Omega$, and $C=10 \mathrm{pF}$ ) versus $L 2\left(V_{\mathrm{in}}=1 \mathrm{~V}, f=50.6 \mathrm{MHz}\right)$.

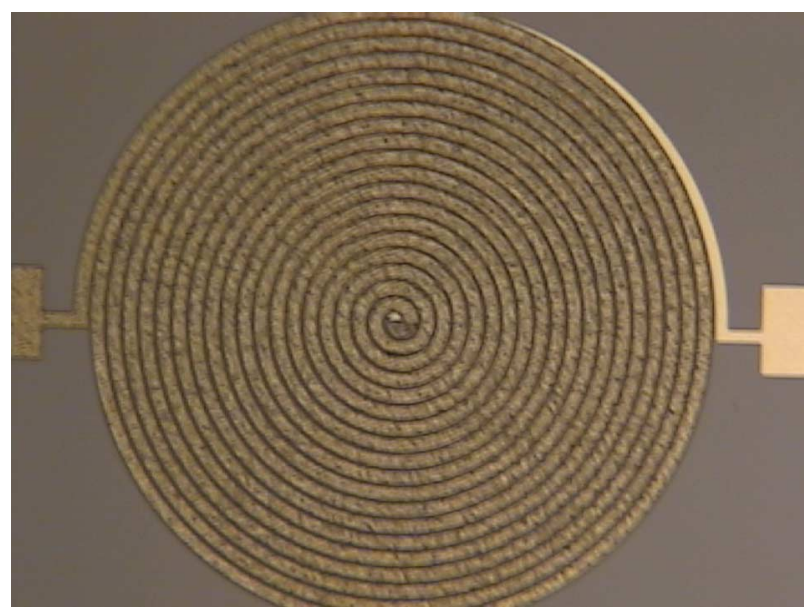

Fig. 5. Microscopic image of the coil fabricated with two metal levels; 19 turns.

(obtaining in this case a value of $S=5.1 \mathrm{mV} / \mathrm{nH}$ ). In addition, the broadening of the peak leads to a wider range of linear behavior of the differential output with respect to $L 2$ (in this case, it is $\Delta L 2 \geq 100 \mathrm{nH}$ ).

\section{Characterization OF THE MICROINDUCTIVE DEVICES}

Microinductive planar coils with a radius of $500 \mu \mathrm{m}$ were fabricated using two metal levels of $\mathrm{Al}$ with 20 (width) $\times$ $1.5 \mu \mathrm{m}$ (height) cross section, each level being formed of by 19 turns. Fig. 5 shows a microscopic image of one of the coils.

To improve the coil sensitivity to the presence of the MPs, a ferromagnetic layer has been deposited in the substrate region of the coil [9], [10]. For this, Si was etched from the back surface of the Si wafers, leaving the coils onto thin Si membranes, and thin Fe layers were grown on the back surface of the membrane using an electrochemical process.

PCB circuit prototypes have been connected with the microinductive devices, using two adjacent integrated inductances as coils $L 1$ and $L 2$. As already indicated in the previous section, 


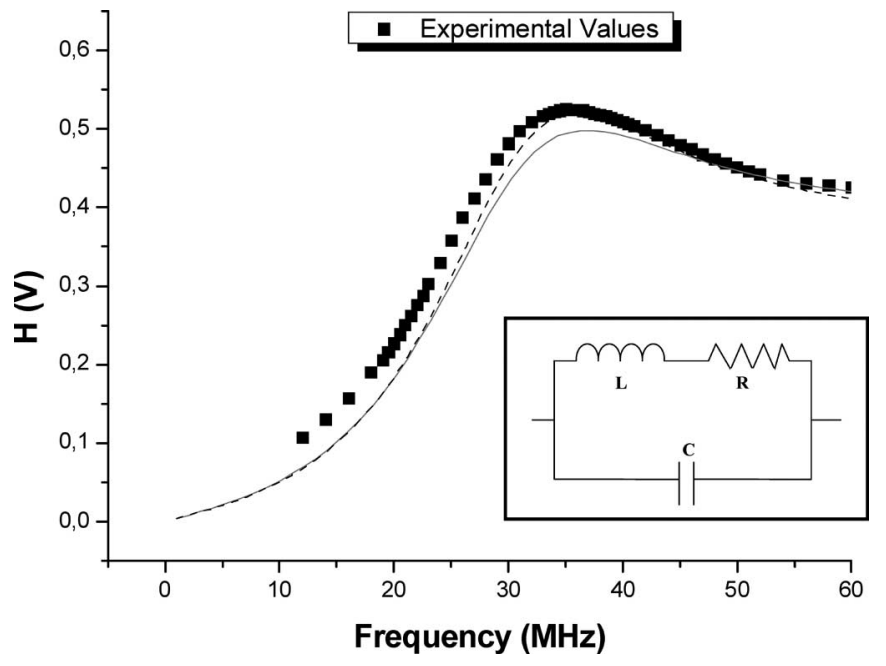

Fig. 6. Experimental results and simulation (PSpice) of the transfer function of a circuit branch with two different equivalent circuits: ( - ) simulation with equivalent circuit shown in inset; (- - - ) simulation with circuit obtained adding a resistance in series with $C$.

$R 1$ and $R 2$ are the serial resistance of the respective coils, and a capacity value of $C=10 \mathrm{pF}$ has been selected in both branches. The transfer function has been measured between 10 and $60 \mathrm{MHz}$.

The results of the measurements performed in one of the circuit branches are plotted in Fig. 6, along with their fitting using the equivalent circuit for the integrated coil shown in the inset of the figure (simulations performed with PSpice).

In this circuit, a capacitance is added in parallel with the inductive and resistive components of the device to model capacitive coupling effects between the metal tracks from the different levels [11]. The full line in the figure has been simulated with $R=62.6 \Omega, L=625 \mathrm{nH}$, and $C=22.4 \mathrm{pF}$. As shown in the figure, this gives a good approximation to the experimental data.

The fitting of these data can be improved by adding to the equivalent circuit a resistance $r$ in series with the capacitance. This resistance models dielectric losses in the insulating layers of the device as well as those determined by induced currents in the substrate [11]. This circuit allows to fit more accurately the experimental transfer function, using $R=60 \Omega, L=690 \mathrm{nH}$, $C=26 \mathrm{pF}$, and $r=55 \Omega$.

Obviously, the introduction of the capacitive component in the equivalent coil circuit drastically modifies the shape of the transfer function. This can be observed in Fig. 7, where the magnitudes of the function calculated without (a) and with (b) the capacitance are compared. As shown in the figure, the presence of the capacitive component induces a decrease in the intensity of the resonant peak and a shift toward lower frequencies.

On the other hand, the comparison of the fitting curves in Fig. 6 reveals that the addition of the resistive component $r$ in series with $C$ does not suppose further significant changes in the shape of the curve. The simulation of the differential output for the circuit configurations corresponding to Fig. 7 leads to sensibility values of $S=10 \mathrm{mV} / \mathrm{nH}$ (a) and $S=0.45 \mathrm{mV} / \mathrm{nH}$ (b), respectively. According to this, the existence of this capacitive component determines a decrease in the sensitivity of the

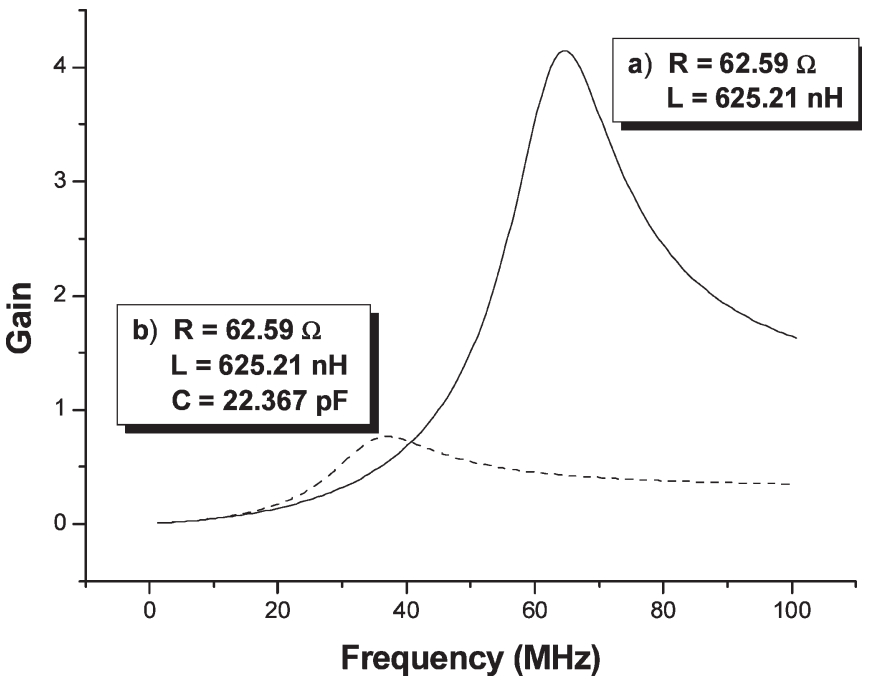

Fig. 7. Magnitude of transfer function of a circuit branch versus frequency, which is calculated without (a) and with (b) a capacitive component in parallel with $L$ and $R$.

differential circuit to the changes of $L 2$, as well as a decrease of the optimum working frequency. These data point out the key role of both $R$ and $C$ parameters as limiting factors of the circuit performance, while $r$ does not seem to have a significant effect. Nevertheless, the obtained sensitivity is still more than one order of magnitude higher than that obtained when working with a nondifferential circuit configuration.

\section{Response to the Presence of MPs: LIMIT OF DETECTION}

The response of the devices has been studied using $2.8-\mu \mathrm{m}$ diameter commercially available Dynabeads M-270 Amine particles [12]. The deposition of the MPs on the device surface is performed using a micropipette, which injects a volume of $0.5 \mu \mathrm{l}$ of a solution with a certain density of MPs over the coil. After evaporation of the solvent (usually deionized water), the MPs remain fixed onto the coil surface. The number of MPs on the surface is determined by counting them from an optical microscopic image.

A previous analysis performed on this kind of device has shown the absence of a quantitative correlation between the inductive changes in the coils and the amount of MPs [9], [10]. This has been related to the complex dependence of the inductance on the distribution of magnetic particles on the coil surface and compromises the ability of these simple planar devices for the quantification of the analyte content. On the other hand, the experimental data show that the coils connected to the differential filter are sensitive to the presence of a minimum density of $2700 \mathrm{MPs} / \mathrm{mm}^{2}$. The measurements of the difference (in magnitude) of the transfer function in both branches of the circuit before and after deposition of the MPs onto the surface of coil $L 2$ are represented in Fig. 8. As shown, the difference of the transfer functions for the coils without MPs gives a nonnull value in the frequency range between 33 and $45 \mathrm{MHz}$

This is due to the existence of slight differences (below 1\%) in the values of the electrical parameters of adjacent coils in the 


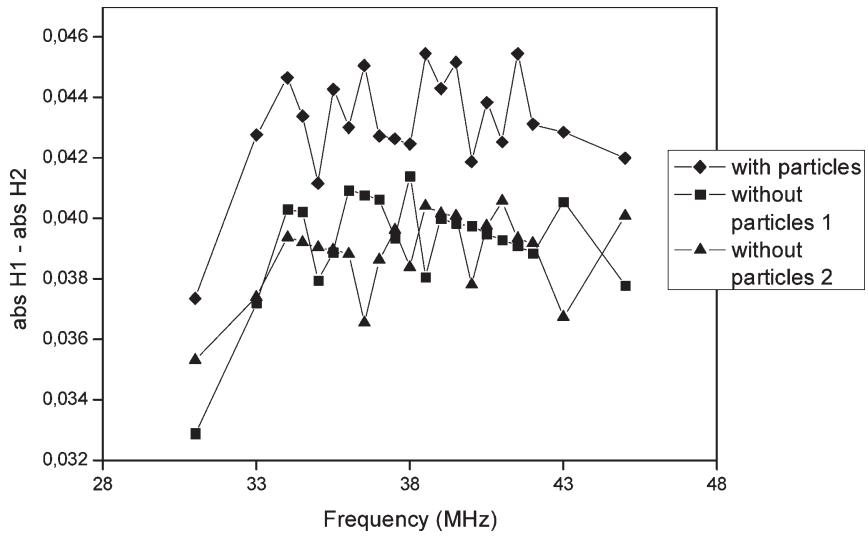

Fig. 8. Difference of transfer function magnitudes measured before and after deposition of $2700 \mathrm{MPs} / \mathrm{mm}^{2}$ onto coil $L 2$.

chips. The figure also indicates some dispersion between the experimental data measured at different times (curves without particles 1 and 2). However, after deposition of the MPs, there is clearly a change in the experimental value of $|H 1|-|H 2|$ which is higher than the experimental uncertainty. This demonstrates the ability of the circuit prototype to react to the presence of this minimum amount of MPs. Taking into account the input voltage used in these measurements $\left(V_{\text {in }} \approx 200 \mathrm{mV}\right)$ and the sensitivity calculated for the differential circuit, the change of inductance related to the output variation observed in the figure is estimated to be of $\Delta L 2 \approx 2 \mathrm{nH}$.

From the detection threshold of $2700 \mathrm{MPs} / \mathrm{mm}^{2}$, a rough estimation of the minimum concentration of analyte can be done, assuming that a minimum of the order of ten molecules of analyte are required to fix a single magnetic particle onto the sensor surface [13]. In addition, there certainly will be molecules that will be fixed on the wrong side of the particle surface (the side away from the active coil surface), and certain amount of molecules may not be involved in the immunological reaction. To take this into account, it is assumed that only $10 \%$ of all the molecules of analyte take part in the fixation of the MPs. This gives a simple estimation of the detection limit of the device in the range of $8 \times 10^{-13} \mathrm{M}$.

\section{CONCLUSION}

In this paper, second-order differential filter circuits working at resonant conditions are proposed for the signal conditioning of microinductive sensor devices. These circuits are characterized by a very high simplicity and have been applied to the characterization of planar microinductive coils aimed at biodetection applications (magnetic immunoassay detectors). The analysis of the circuits gives a sensitivity of the differential output in the range of $S=0.45 \mathrm{mV} / \mathrm{nH}$, which is limited by the series resistance and the parasitic capacitive component of the microinductive devices. However, in spite of these effects, this value is more than one order of magnitude higher than that obtained when working with similar circuits with nondifferential configurations. Experimentally, this has allowed the detection of changes down to $2 \mathrm{nH}$, which allows the detection of a minimum MP density of $2700 \mathrm{MPs} / \mathrm{mm}^{2}$, corresponding to a detection limit of the analyte below $1 \mathrm{pM}$. Optimization of the coils in terms of both resistance and capacitance components would allow a sensitivity improvement of almost four orders of magnitude, with a simulated sensitivity for the differential circuits in the range of $300 \mathrm{mV} / \mathrm{nH}$. These data corroborate the viability of the extremely simple differential circuits for the signal conditioning of microinductive devices for very highsensitivity applications.

\section{REFERENCES}

[1] K. Larsson, K. Kriz, and D. Kriz, "Magnetic transducers in biosensors and bioassays," Analysis, vol. 27, no. 7, pp. 617-621, 1999.

[2] D. L. Graham, H. A. Ferreira, and P. P. Freitas, "Magnetoresistive-based biosensors and biochips," Trends Biotechnol., vol. 22, no. 9, pp. 455-462, Sep. 2004.

[3] M. A. M. Gijs, "Magnetic bead handling on-chip: New opportunities for analytical applications," Microfluid Nanofluid, vol. 1, no. 1, pp. 22-40, Nov. 2004.

[4] C. B. Kriz, K. Radevik, and D. Kriz, "Magnetic permeability measurements in bioanalysis and biosensors," Anal. Chem., vol. 68, no. 11, pp. 1966-1970, 1996.

[5] J. Richardson, P. Hawkins, and R. Luxton, "The use of coated paramagnetic particles as a physical label in a magneto-immunoassay," Biosens. Bioelectron., vol. 16, no. 9-12, pp. 989-993, Dec. 2001.

[6] J. Richardson, A. Hill, R. Luxton, and P. Hawkins, "A novel measuring system for the determination of paramagnetic particle labels for use in magneto-immunoassays," Biosens. Bioelectron., vol. 16, no. 9-12, pp. 1127-1132, Dec. 2001.

[7] K. Diaz de Lezama, A. García-Arribas, J. M. Barandiarán, and J. Gutiérrez, "Comparative study of alternative circuit configurations for inductive sensors," Sens. Actuators A, Phys., vol. 91, no. 1/2, pp. 226-229, Jun. 2001.

[8] S. Baglio, S. Castorina, and N. Savalli, "Strategies and circuits for micro inductive biosensor conditioning," in Proc. Eurosensors XVI: 16th Eur. Conf. Solid-State Transducers, Prague, Czech Republic, Sep. 15-18, 2002, pp. 604-607.

[9] S. Martínez, "Desenvolupament de dispositius microinductius en tecnologia de Si per a aplicacions de biodetecció a molt alta sensibilitat," Ph.D. dissertation, Univ. Barcelona, Barcelona, Spain, 2004.

[10] C. Serre, S. Martínez, A. Pérez-Rodríguez, J. R. Morante, J. Esteve, and J. Montserrat, "Si technology based microinductive devices for biodetection applications," Sens. Actuators A, Phys., vol. 132, no. 2, pp. 499-505, 2006.

[11] W. G. Hurley, M. C. Duffy, S. O’Reilly, and S. C. O’Mathuana, "Impedance formulas for planar magnetic structures with spiral windings," IEEE Trans. Ind. Electron., vol. 46, no. 2, pp. 271-278, Apr. 1999.

[12] Dynal Biotech. [Online]. Available: http://www.dynal.no

[13] Bangs Laboratories Inc., Technote \#301: Immunological Applications. [Online]. Available: http://www.bangslab.com

[14] PSpice: Standard Commercial Circuit Simulator, From Orcad, Cadence Design Systems, Inc. [Online]. Available: http://www.orcad.com/

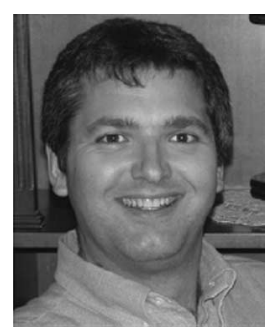

Salvatore Baglio was born in Catania, Italy, in 1965. He received the Laurea and $\mathrm{Ph} . \mathrm{D}$. degrees from the University of Catania in 1990 and 1994, respectively.

He was a Lecturer of automatic control theory at the University of Messina, Messina, Italy, and of electronic measurement systems at the University of Catania. He was also a Consultant for STMicroelectronics in the field of soft computing methodologies for nonlinear and chaotic circuits and systems. Since 1996, he has been with the Dipartimento di Ingegneria Elettrica Elettronica e dei Sistemi, University of Catania, where he is currently Associate Professor of electronic instrumentation and measurements. He teaches courses in measurement theory and sensors and transducers. He is the coauthor of more than 150 scientific publications, among which are papers that have been published in international journals, presented at international conferences, or appeared as chapters in books, and he is the holder of several U.S. patents. His research interests are mainly focused on measurement methodologies, smart sensors, microsensors, and microsystems.

Dr. Baglio has been an Associate Editor for the IEEE TRANSACTIONS ON CIRCUITS AND SYSTEMS and a distinguished Lecturer for the IEEE Circuits and System Society. 


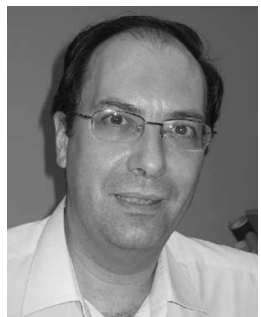

Alejandro Pérez-Rodríguez was born in 1961 in San Cristóbal, Venezuela. He received the first degree and the Ph.D. degree (with Doctorate Award from the university) in physics from the University of Barcelona, Barcelona, Spain, in 1984 and 1987, respectively.

In 1985, he was with the Department of Applied Physics and Electronics, University of Barcelona, as Associated Lecturer, where he has been Permanent Professor ("Profesor Titular") in the Department of Electronics since 1988. During 1988-1989, he was a Visiting Research Fellow at the University of Surrey, Surrey, U.K. Since December 2004, he has been Vice-Dean of the Faculty of Physics, University of Barcelona. He does research at the Laboratory of Electronic Materials and Engineering, where he coordinates research projects related to Si microsystem technologies, ion beam processing of nanostructured materials in group IV technologies, and advanced characterization and processes for Si-based micromachining technologies. He is a coauthor of 100 papers in the main refereed scientific journals in these fields, as well as more than 200 contributions in international conferences (ten as invited)

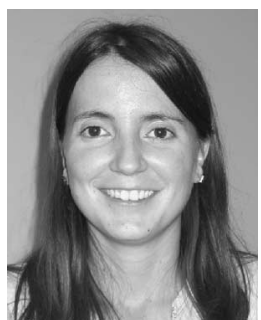

Susanna Martínez received the degree in physics and the Ph.D. degree from the University of Barcelona, Barcelona, Spain, in 1999 and 2004, respectively.

Since 1999, she has been with the Electronics Department, University of Barcelona, as a Research and Teaching Assistant. Her principal research interests include signal processing and data acquisition for the characterization of magnetic devices and simulation, design, fabrication, and characterization of high-sensitivity magnetic biosensors and microfuel cells in microelectromechanical system technology.

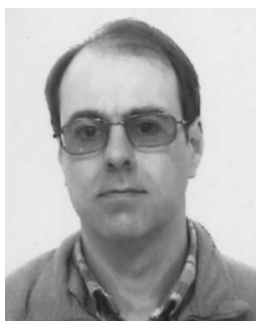

Christophe Serre was born in Tarbes, France, in 1963. He received the Ph.D. degree in microelectronics from the University of Grenoble, Grenoble, France, in 1992.

During 1993, he was an Assistant Professor at the University of Grenoble, where he worked on the luminescence of porous silicon at the Laboratory of Physical Spectrometry. He was with the Electronic Materials and Engineering Laboratory, Department of Applied Physics and Electronics, University of Barcelona, Barcelona, Spain, in November 1993 as a Postdoctoral Fellow. Since February 2002, he has been working as a Researcher in the same laboratory, within the "Ramon y Cajal" program. His research activities focus on the fabrication and characterization of $\mathrm{SiC}$ and $\mathrm{SiGeC}$ thin films for micromechanics as well as micromachining technologies and their application to integrated sensors and actuators.

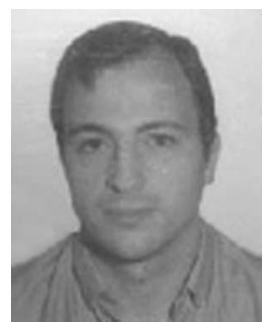

Joan Ramon Morante was born in Mataró, Spain He received the Ph.D. degree in physics from the University of Barcelona, Barcelona, Spain, in 1980.

Since 1986, he has been Full Professor of electronics and Director of the Electronic Materials and Engineering Group, Electronic Materials and Engineering, University of Barcelona. He has been Dean and Vice-Dean of the Physics Faculty, Academic Advisor of the electronic engineering degree, and Director of the Department of Electronics, which is associated with the National Microelectronics Centre, Bellaterra, Barcelona. He is Codirector of the Centre for Engineering of Microsystems and Director of the Centre of the Reference of Advanced Materials for Energy. His activity is devoted to the electronic materials and technology, physics and chemical sensors, actuators, and microsystems. He has special interest in nanoscience, micro and nanotechnologies, advanced nanomaterials for energy, and integrated sensors and microsystems applications. $\mathrm{He}$ has collaborated in international R\&D projects such as BRITE, GROWTH, priority 3 (micromechanics, microengineering, gas sensors, nanomaterials, nanotechnologies,...), ESPRIT, IST, priority 2 (advanced devices, sensors, actuators, microsystems, electronic systems,...), JOULE,..., and industrial projects such as EUREKA, IBEROEKA, and CRAFT's, as well as industrial private contracts. He is the coauthor of more than 400 papers in international specialized journals and a member of international committees and editorial boards in the field of electronic materials and micro-nanotechnology, sensors and actuators, and microsystems.

Dr. Morante received the Research Prize Narciso Monturiol from the Generalitat of Catalunya, Spain, and the "Senior Research Distinction" award from the Generalitat of Catalunya, Spain.

Jaume Esteve received the Ph.D. degree in physical electronics from the University of Barcelona, Barcelona, Spain, in 1988.

During 1984 and 1985, he performed research with the Applied Physics Department, University of Barcelona. In 1985, he was with the Centre Nacional de Microelectrònica (CNM)-CSIC, Bellatera, Spain, where he worked in metallization processes. During 1989, he was a Visiting Researcher at the Interuniversitair Micro-Elektronic Centrum, Leuven, Belgium. In 1990, he was with the Department of Silicon Technology and Microsystems, CNM, as a Senior Researcher Scientist. His areas of interest are silicon micromachining technologies and their application to integrated sensors and actuators. He has participated and has been project leader of different national and European projects in this field.

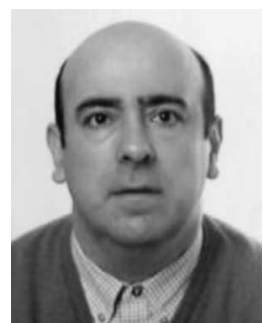

Josep Montserrat received the B.S. and Ph.D. degrees in physics from the University of Barcelona, Barcelona, Spain, in 1985 and 1991, respectively.

In 1987, he was with the National Microelectronics Centre, Bellaterra, Spain. He works as a Process Engineer in the Clean Room Group. He is responsible for ion implantation and metallization areas. His main research interest is in silicon technology for the manufacture of CMOS integrated circuits, power devices, and microelectronic sensors. 